\title{
Study of PIK3CA, BRAF, and KRAS mutations in breast carcinomas among Chinese women in Qinghai
}

\author{
Y.L. Wang ${ }^{1}$, X. Dai' ${ }^{1}$, Y.D. Liं ${ }^{2}$, R.X. Cheng ${ }^{1}$, B. Deng ${ }^{1}$, X.X. Geng ${ }^{1}$ and H.J. Zhang ${ }^{1}$ \\ ${ }^{1}$ College of Life and Science, Huaibei Normal University, Huaibei, China \\ ${ }^{2}$ Biological Resources in Qinghai, School of Medicine, Qinghai University, Xining, China
}

Corresponding author: H.J. Zhang

E-mail: haijunzhang@163.com

Genet. Mol. Res. 14 (4): 14840-14846 (2015)

Received June 11, 2015

Accepted September 23, 2015

Published November 18, 2015

DOI http://dx.doi.org/10.4238/2015.November.18.49

\begin{abstract}
Phosphatidylinositol-3-OH kinase and RAS-activated signaling pathways play an important role in tumor formation. Abnormalities in relevant genes play essential roles in the occurrence and development of many human cancers. Studies of breast cancer have mainly focused on the women in western countries, but few studies have examined the frequency of mutations in PIK3CA, BRAF, and KRAS in Chinese breast cancer patients. In this study, we conducted sequence analysis of PIK3CA, $B R A F$, and KRAS and determined relationships with the occurrence of breast cancer in women from Qinghai. DNA was extracted from 25 cases of human breast cancer tissue samples. PIK3CA, BRAF, and KRAS mutation analysis was performed by polymerase chain reaction and DNA sequencing. No mutations were found in PIK3CA, BRAF, and KRAS of adjacent tissues. However, PIK3CA mutations were observed in $32 \%$ (8) of the 25 breast cancer tissues examined, in which exon 9 accounted for $4 \%$ (1), exon 20 accounted for $28 \%$ (7), and no mutations were found in exon 1 of PIK3CA. Sequencing of exon 2 of KRAS suggested that $20 \%$ (5) of the 25 samples harbored a mutation and $16 \%$ (4) of BRAF
\end{abstract}


harbored a mutation. Any mutation in these 3 oncogenes may induce the occurrence and development of breast cancer.

Key words: BRAF; Breast cancer; KRAS; Mutation; PIK3CA

\section{INTRODUCTION}

Breast cancer is one of the most common malignant tumors. In women, breast cancer is the most frequent causes of cancer death (Lai et al., 2008). The occurrence and development of breast cancer are complicated processes involving multiple factors and steps resulting from a series of gene alterations.

PIK3CA plays a vital role in coding $\mathrm{P} 110$ catalytic subunit of the class I phosphatidylinositol3-kinases (PI3K). PIK3CA mutations result in activation of the PI3K/AKT signaling pathway, which regulates cell metabolism, growth, proliferation, and survival (Osaki et al., 2004; Workman, 2004; Board et al., 2008; Lin et al., 2009). PIK3CA mutations occur mainly in 2 hotspots, including the kinase domain from exon 20 and helical domain from exon 9 , as well as occasionally in exon 1 (Board et al., 2008; Simi et al., 2008; Stemke-Hale et al., 2008).

The BRAF gene is frequently activated in the mitogen-activated protein kinase/extracellular signal-regulated kinase signaling pathway, which affects cell division and differentiation (Feng et al., 2005). Studies have showed that the BRAF gene encodes for the 32-kDa BRAF protein composed of 783 amino acids (Fransén et al., 2004; Kim et al., 2006). The KRAS protein is also an essential regulatory factor upstream of the mitogen-activated protein kinase/extracellular signal-regulated kinase pathway (Ji et al., 2007). Although BRAF and KRAS are both involved in the signaling pathway, mutations in their 2 genes were found to be mutually exclusive (Ahlquist et al., 2008). The cellular and molecular mechanisms conferring oncogenic effects and activating KRAS mutations remain incompletely understood (Simi et al., 2008). Studies have showed that activation of the mitogen-activated protein kinase pathway was important for the development of colorectal cancer via BRAF or KRAS mutations (Popescu et al., 1985). Thus, in this study, we examined whether the $B R A F$ and $K R A S$ gene have the same effect in breast cancer.

We detected mutations of PIK3CA, BRAF, and KRAS in 25 breast cancer tissues to determine whether the mutations were related to the occurrence of breast cancer.

\section{MATERIAL AND METHODS}

\section{Samples}

Breast cancer tissues samples and the corresponding adjacent tissues were collected from 25 patients, and all cases were confirmed by pathological diagnosis. Tissue samples were conserved in $-20^{\circ} \mathrm{C}$.

\section{Polymerase chain reaction (PCR) amplification and sequencing}

Because PIK3CA gene mutations in human cancers are frequently observed in exons 9 and 20, and occasionally in exon 1, we focused on these 3 exons in PIK3CA. Additionally, 
primers were designed to detect mutations in exon 2 of KRAS and exon 15 of BRAF. The primers used for sequencing PIK3CA, BRAF, and KRAS (are shown in Table 1). PCR was carried out to amplify fragments of these 3 genes. PCR was conducted in $25-\mu \mathrm{L}$ volume containing: $2.5 \mu \mathrm{L}$ PCR buffer (Takara, Shiga, Japan), $4 \mu \mathrm{L} 2.5 \mathrm{mM}$ dNTPs, $2 \mu \mathrm{L} 25 \mathrm{mM} \mathrm{MgCl}, 0.4 \mu \mathrm{L} 10 \mu \mathrm{M}$ of each forward and reverse primer (Generay Bioengineering, Shanghai, China), $0.25 \mu \mathrm{L}$ rTaq DNA polymerase. PCR was performed at $95^{\circ} \mathrm{C}$ for $5 \mathrm{~min}$, followed by 35 cycles of $95^{\circ} \mathrm{C}$ for $30 \mathrm{~s}, 55^{\circ} \mathrm{C}$ for $30 \mathrm{~s}$ and $72^{\circ} \mathrm{C}$ for $30 \mathrm{~s}$, with a final extension for $10 \mathrm{~min}$. The PCR products were evaluated by $1.5 \%$ agarose gel electrophoresis, dyed with ethidium bromide, and visualized under UV light using a gel-imager. PCR products were purified using a PCR Purification kit (Invitrogen). The purified products were sequenced in both directions using an ABI 3730 (Applied Biosystems, Foster City, CA, USA).

\section{Table 1. List of primers and PCR conditions used in this study.}

\begin{tabular}{|c|c|c|c|}
\hline Gene & Primer sequence $\left(5^{\prime} \rightarrow 3^{\prime}\right)$ & Annealing temperature & Fragment \\
\hline \multicolumn{4}{|l|}{ PIКЗCA } \\
\hline Exon 20 & $\begin{array}{l}\text { F: CAGGAGATGTGTTACAAGGCTTAT } \\
\text { R: TCAGTTCAATGCATGCTGTTTTAAT }\end{array}$ & $55^{\circ}$ & 267 \\
\hline Exon 9 & $\begin{array}{l}\text { F: AGTAACAGACTAGCTAGAGACAAT } \\
\text { R: CCATTTTAGCACTTACCTGTGAC }\end{array}$ & $55^{\circ}$ & 141 \\
\hline Exon 1 & $\begin{array}{l}\text { F: CTCCACGACCATCATCAGG } \\
\text { R: GATTACGAAGGTATTGGTTTAGACAG }\end{array}$ & $55^{\circ}$ & 420 \\
\hline \multicolumn{4}{|c|}{ 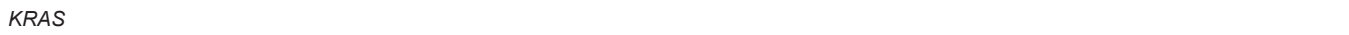 } \\
\hline Exon 2 & $\begin{array}{l}\text { F: AGGCCTGCTGAAAATGACTGAA } \\
\text { R: AAAGAATGGTCCTGCACCAG }\end{array}$ & $55^{\circ}$ & 168 \\
\hline \multicolumn{4}{|l|}{$B R A F$} \\
\hline Exon 15 & $\begin{array}{l}\text { F: AATGCTTGCTCTGATAGGAAAA } \\
\text { R: AGCATCTCCAGCGCCAAAAAT }\end{array}$ & $55^{\circ}$ & 230 \\
\hline
\end{tabular}

\section{RESULTS}

We analyzed 25 human breast cancer tissues to detect mutations in PIK3CA, BRAF, and KRAS genes by PCR amplification and direct bilateral sequencing (Table 2). The results showed that PIK3CA mutations (exons 20, 9, and 1) were detected in 32\% (8) of the 25 patients with breast cancer, while 28 and $4 \%$ of mutations were in exons 20 and 9 , respectively. No mutation was found in exon 1 of the 25 samples, but in the intron, we indetified a mutation in A390G. Mutations in PIK3CA were located in 2 primary hotspots $(1633 \mathrm{G} \rightarrow \mathrm{A}, 3140 \mathrm{~A} \rightarrow \mathrm{G}), B R A F$ in $1808(\mathrm{G} \rightarrow \mathrm{C})$ and $K R A S$ in $100(\mathrm{C} \rightarrow \mathrm{A})$. The frequencies of BRAF and KRAS mutations were 16 and 20\% (Table 2), respectively. Moreover, 2 mutant sites, $3140(A \rightarrow G)$ of $P I K 3 C A$ and $100(C \rightarrow A)$ of $K R A S$, coexisted. The $1808(\mathrm{G} \rightarrow \mathrm{C})$ mutations in $B R A F$ and the $100(\mathrm{C} \rightarrow \mathrm{A})$ mutations in $K R A S$ have not been reported previously in breast cancer. All mutations in these 3 genes were in cases of invasive ductal cancer. Previous studies suggested that mutations in KRAS, BRAF, and PIK3CA were frequently observed in several types of human cancers, including colon, breast, and lung cancers. However, mutual mutations in these 3 genes in different types of cancer have not been thoroughly investigated. Xu et al. (2011) found that only $5.9 \%$ of mutations were in both PIK3CA and KRAS in human cancer of cholangiocarcinoma. In our study, we found that PIK3CA mutations coexisted with KRAS mutations in one sample. We unexpectedly detected mutual mutations of $3140 \mathrm{~A}-\mathrm{G}$ and 3134A-C in PIK3CA (Figure 1). 


\begin{tabular}{|c|c|c|c|}
\hline \multirow[t]{2}{*}{ Sample/test result } & \multicolumn{3}{|c|}{ Mutation result } \\
\hline & PIK3CA nucleotide change & $B R A F$ nucleotide change & $K R A S$ nucleotide change \\
\hline 1 Mutation detected & None & None & Exon $2100 \mathrm{C} \rightarrow \mathrm{A}$ \\
\hline 2 Mutation detected & None & None & None \\
\hline 3 Mutation detected & None & None & Exon $2100 C \rightarrow A$ \\
\hline 4 Mutation detected & Exon $203140 A \rightarrow G$ & Exon $151808 \mathrm{G} \rightarrow \mathrm{C}$ & None \\
\hline 5 Mutation detected & Exon $203140 \mathrm{~A} \rightarrow \mathrm{G}$ & None & None \\
\hline 6 Mutation detected & Exon $203140 A \rightarrow G$ & Exon $151808 \mathrm{G} \rightarrow \mathrm{C}$ & None \\
\hline 7 Mutation not detected & - & - & - \\
\hline 8 Mutation detected & Exon $203140 \mathrm{~A} \rightarrow \mathrm{G}$ & None & None \\
\hline 9 Mutation detected & Exon 9 1633G $\rightarrow \mathrm{A}$ & Exon $151808 \mathrm{G} \rightarrow \mathrm{C}$ & None \\
\hline 10 Mutation not detected & - & - & - \\
\hline 11 Mutation not detected & - & - & - \\
\hline 12 Mutation detected & Exon $203140 \mathrm{~A} \rightarrow \mathrm{G}$ & None & None \\
\hline 13 Mutation detected & Exon $203140 \mathrm{~A} \rightarrow \mathrm{G}$ & None & Exon $2100 C \rightarrow A$ \\
\hline 14 Mutation not detected & - & - & - \\
\hline 15 Mutation detected & None & None & Exon $2100 \mathrm{C} \rightarrow \mathrm{A}$ \\
\hline 16 Mutation detected & None & Exon $151808 \mathrm{G} \rightarrow \mathrm{C}$ & None \\
\hline 17 Mutation not detected & - & - & - \\
\hline 18 Mutation detected & None & None & Exon $2100 \mathrm{C} \rightarrow \mathrm{A}$ \\
\hline 19 Mutation not detected & - & - & - \\
\hline 20 Mutation not detected & - & - & - \\
\hline 21 Mutation not detected & - & - & - \\
\hline 22 Mutation not detected & - & - & - \\
\hline 23 Mutation not detected & - & - & - \\
\hline 24 Mutation not detected & - & - & - \\
\hline 25 Mutation detected & Exon $203140 \mathrm{~A} \rightarrow \mathrm{G}$ & - & None \\
\hline Mutated (\%) & $8(32 \%)$ & $4(16 \%)$ & $5(20 \%)$ \\
\hline
\end{tabular}

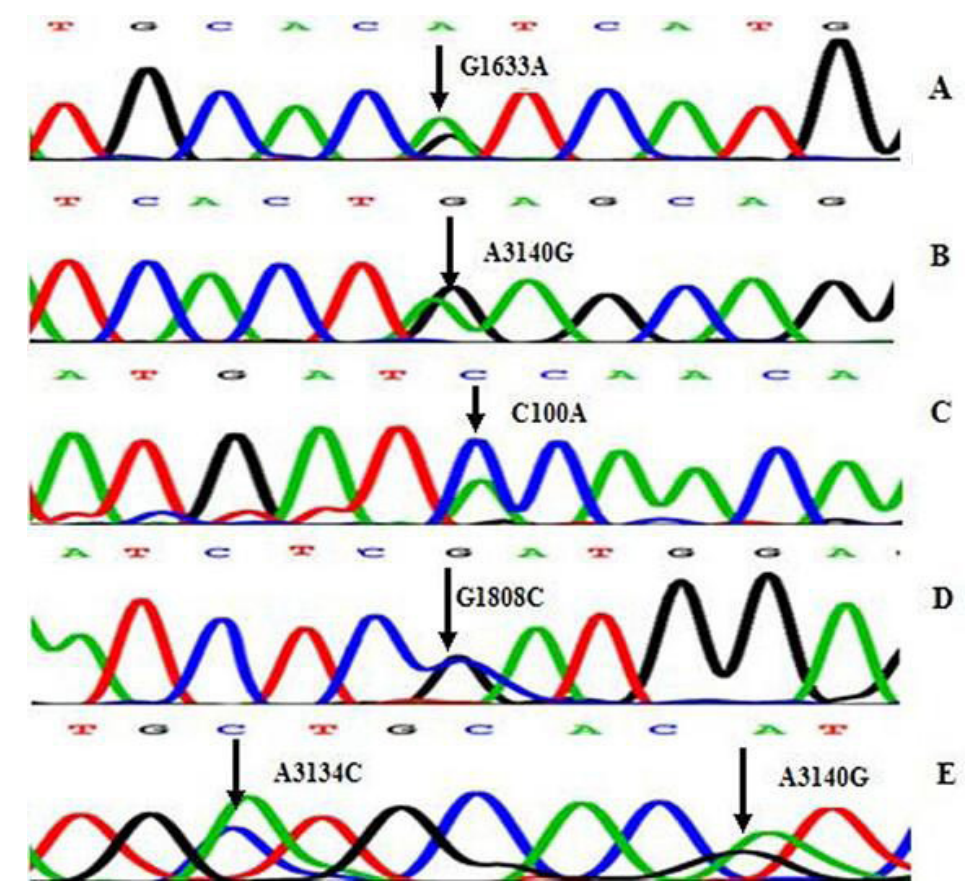

Figure 1. Results of direct sequencing of PIK3CA in exon 9 (A) and exon 20 (B), BRAF in exon 15 (D) and KRAS in exon 2 (C). A3134C and A3140G are mutual mutations in PIK3CA (E). 


\section{DISCUSSION}

Most previously published studies concerning simultaneous PIK3CA, KRAS, or BRAF mutations were concentrated on colorectal cancer (De Roock et al., 2010). Some studies suggested that KRAS and PIK3CA mutations coexisted within the same tumor (Velho et al., 2005; Siena et al., 2009; De Roock et al., 2010). PIK3CA mutations were coincident with RAS pathway mutations in colorectal cancers (Siena et al., 2009), whereas a previous study found that mutational activation of the PI3K pathway was mutually exclusive with the RAS pathway in breast cancer (Ahlquist et al., 2008).

In this study, we found that frequency of PIK3CA, BRAF, and KRAS mutations were in 32, 16 , and $20 \%$, respectively, in breast cancer patients. PIK3CA mutations occurred more frequently in conjunction with KRAS or BRAF mutations than in PIK3CA alone, suggesting a possible synergistic effect in the signaling pathways controlled by the oncogenes in colorectal cancer (Velho et al., 2005; Nosho et al., 2008). In 52\% of all patients, at least one mutation of these 3 genes was found in the research of breast cancer. Both the mutant locations in PIK3CA and the mutation frequency were very similar to previous studies in Caucasian breast cancer (Saal et al., 2005). Regarding histologic types, the mutant types of PIK3CA in breast cancer have rarely been reported other than in invasive ductal carcinomas (Lin et al., 2009). Two mutational hotspots (exons 9 and 20) of PIK3CA have been identified in various malignancies, including breast, lung, and colorectal cancers (Samuels et al., 2004).

In different tumor types, activated key signals of the PI3K/protein kinase B (AKT)/ mammalian target of rapamycin and RAS/RAF/MEK signaling pathways regulate cell proliferation, growth, apoptosis, invasion, and migration (Peyssonnaux and Eychène, 2001; Engelman, 2009). Several studies have suggested that aberrations in PI3K/AKT/mammalian target of rapamycin and the MAP kinase pathway likely coexist, but few studies have examined this, and most were concentrated on colorectal cancer (Engelman, 2009; Ihle et al., 2009). PIK3CA expression is directly associated with the expression of epidermal receptor growth factors family members (EGFR and human epidermal growth factor receptor 2). Currently, numerous events are mediated by the overexpression of EGFR and human epidermal growth factor receptor 2, which induce an increase in the PI3K/Akt signaling pathway (Thompson and Thompson, 2004). KRAS is a downstream regulated gene and plays a vital role in the EGFR signal transduction pathway. However, KRAS mutation causing EGFR signals to not be accepted may automatically activate the pathway and downstream signals (Soulières et al., 2010). Not all patients could be tested for mutations because of the limited amount of tumor tissues available. Mutations in KRAS and BRAF were previously found to be mutually exclusive (Davies et al., 2002). Additionally, activation of the RAS/RAF/MEK pathway mediated the resistance to PI3K inhibitors of PIK3CA mutation in tumors (Ihle et al., 2009; Janku et al., 2012). Clinical data have shown that a single PI3K/AKT/mammalian target of rapamycin pathway inhibitor may be insufficient for inducing a response, as PIK3CA mutations often coexist with other concurrent molecular aberrations (Ihle et al., 2009; Di Nicolantonio et al., 2010). Mutations in PIK3CA, BRAF, and KRAS may trigger sustained activation of the RAS/RAF/ MAPK and PI3K/PTEN/Akt signaling pathways.

In conclusion, bidirectional sequencing was used to detect mutations in 3 oncogenes. Gene mutations and activation of molecular pathways play a vital role in tumor formation. Therefore, our results are important for studies aimed at improving the treatment of breast cancer. 


\section{Conflicts of interest}

The authors declare no conflict of interest.

\section{ACKNOWLEDGMENTS}

Research supported by the National Natural Science Foundation of China (\#81272377), the Anhui Provincial Natural Science Foundation (\#1208085 MC45), and the Natural Science Key Foundation (\#KJ2010A299) from Department of Education, Anhui Province. We thank all patients for agreeing to provide samples. We are also indebted to all members of our laboratory for their help in performing this research.

\section{REFERENCES}

Ahlquist T, Bottillo I, Danielsen SA, Meling GI, et al. (2008). RAS signaling in colorectal carcinomas through alteration of RAS, RAF, NF1, and/or RASSF1A. Neoplasia 10: 680-686.

Board RE, Thelwell NJ, Ravetto PF, Little S, et al. (2008). Multiplexed assays for detection of mutations in PIK3CA. Clin. Chem. 54: 757-760.

Davies H, Bignell GR, Cox C, Stephens P, et al. (2002). Mutations of the BRAF gene in human cancer. Nature 417: 949-954.

De Roock W, Claes B, Bernasconi D, De Schutter J, et al. (2010). Effects of KRAS, BRAF, NRAS, and PIK3CA mutations on the efficacy of cetuximab plus chemotherapy in chemotherapy-refractory metastatic colorectal cancer: a retrospective consortium analysis. Lancet Oncol. 11: 753-762.

Di Nicolantonio F, Arena S, Tabernero J, Grosso S, et al. (2010). Deregulation of the PI3K and KRAS signaling pathways in human cancer cells determines their response to everolimus. J. Clin. Invest. 120: 2858- 2866.

Engelman JA (2009). Targeting PI3K signaling in cancer: opportunities, challenges and limitations. Nat. Rev. Cancer 9: 550-562.

Feng YZ, Shiozawa T, Miyamoto T, Kashima H, et al. (2005). BRAF mutation in endometrial carcinoma and hyperplasia: correlation with KRAS and p53 mutations and mismatch repair protein expression. Clinical Cancer Res. 11: 6133-6138.

Fransén K, Klintenãs M, Osterström A, Dimberg J, et al. (2004). Mutation analysis of the BRAF, ARAF and RAF-1 genes in human colorectal adenocarcinomas. Carcinogenesis 25: 527-533.

Ihle NT, Lemos R Jr, Wipf P, Yacuob A, et al. (2009). Mutations in the phosphatidylinositol-3-kinase pathway predict for antitumor activity of the inhibitor PX-866 whereas oncogenic Ras is a dominant predictor for resistance. Cancer Res. 69: 143-150.

Janku F, Wheler JJ, Westin SN, Moulder SL, et al. (2012). PI3K/AKT/mTOR inhibitors in patients with breast and gynecologic malignancies harboring PIK3CA mutations. J. Clin. Invest. 30: 777-782.

Ji H, Wang ZX, Perera SA, Li D, et al. (2007). Mutations in BRAF and KRAS converge on activation of the mitogen-activated protein kinase pathway in lung cancer mouse models. Cancer Res. 67: 4933-4939.

Kim IJ, Kang HC, Jang SG, Kim K, et al. (2006). Oligonucleotide microarray analysis of distinct gene expression patterns in colorectal cancer tissues harboring BRAF and KRAS mutations. Carcinogenesis 27: 392-404.

Lai YL, Mau BL, Cheng WH, Chen HM, et al. (2008). PIK3CA exon20 mutation is independently associated with a poor prognosis in breast cancer patients. Ann. Surg. Oncol. 15: 1064-1069.

Lin Y, Jiang XF, Shen Y, Li M, et al. (2009). Frequent mutations and amplifications of the PIK3CA gene in pituitary tumors. Endocr. Relat. Cancer 16: 301-310.

Nosho K, Irahara N, Shima K, Kure S, et al. (2008). Comprehensive biostatistical analysis of CpG island methylator phenotype in colorectal cancer using a large population-based sample. PLOS One 3: e3698.

Osaki M, Oshimura M and Ito H (2004). PI3K-AKT pathway: its functions and alterations in human cancer. Apoptosis 9: 667-676.

Peyssonnaux C and Eychène A (2001). The Raf/MEK/ERK pathway: new concepts of activation. Biol. Cell 93: 53-62.

Popescu NC, Amsbaugh SC, DiPaolo JA, Tronick SR, et al. (1985). Chromosomal localization of three human ras genes by in situ molecular hybridization. Somat. Cell Mol. Genet. 11: 149-155.

Saal LH, Holm K, Maurer M, Memeo L, et al. (2005). PIK3CA mutations correlate with hormone receptors, node metastasis, and ERBB2, and are mutually exclusive with PTEN loss in human breast carcinoma. Cancer Res. 65: 2554-2559.

Samuels Y, Wang Z, Bardelli A, Silliman N, et al. (2004). High frequency of mutations of the PIK3CA gene in human cancers. Science 304: 554.

Siena S, Sartore-Bianchi A, Di Nicolantonio F, Balgour J, et al. (2009). Biomarkers predicting clinical outcome of epidermal growth factor receptor-targeted therapy in metastatic colorectal cancer. J. Natl. Cancer Inst. 101: 1308-1324. 
Simi L, Pratesi N, Vignoli M, Sestini R, et al. (2008). High-resolution melting analysis for rapid detection of KRAS, BRAF and PIK3CA gene mutations in colorectal cancer. Am. J. Clin. Pathol. 130: 247-253.

Soulières D, Greer W, Magliocco AM, Huntsman D, et al. (2010). KRAS mutation testing in the treatment of metastatic colorectal cancer with anti-EGFR therapies. Curr. Oncol. 17: 31-40.

Stemke-Hale K, Gonzalez-Angulo AM, Lluch A, Neve RM, et al. (2008). An integrative genomic and proteomic analysis of PIK3CA, PTEN, and AKT mutations in breast cancer. Cancer Res. 68: 6084-6091.

Thompson JE and Thompson CB (2004). Putting the rap on Akt. J. Clin. Oncol. 22: 4217-4226.

Velho S, Oliveira C, Ferreira A, Ferreira AC, et al. (2005). The prevalence of PIK3CA mutations in gastric and colon cancer. Eur. J. Cancer 41: 1649-1654.

Workman P (2004). Inhibiting the phosphoinositide3-kinase pathway for cancer treatment. Biochem. Soc. Trans. 32: 393-396. Xu RF, Sun JP, Zhang SR, Zhu GS, et al. (2011). KRAS and PIK3CA but not BRAF genes are frequently mutated in Chinese cholangiocarcinoma patients. Biomed. Pharmacother. 65: 22-26. 